\title{
An Overview of the Effect of Diet on the Allergenicity of Cats to Susceptible Humans
}

\author{
Julia Guazzelli Pezzali ${ }^{1}$, Spencer C Smith ${ }^{1}$ and Charles G Aldrich ${ }^{*}$
}

${ }^{1}$ Grain Science and Industry Department, Kansas State University, Manhattan, KS, USA

Received: 27 January, 2018; Accepted: 8 February, 2018; Published: 12 February, 2018

*Corresponding author: Charles G Aldrich, Kansas State University, 201 Shellenberger Hall, Manhattan, KS, USA, Tel: +785-532-6199; E-mail: aldrich4@ksu.edu

\begin{abstract}
Relationships between humans and their pets have evolved to the point that pets are considered members of the family. Cats are one of the most popular pets worldwide, but many people suffer from allergies to pet dander. As a result, allergies are the second leading cause for cat relinquishment. Cat dander consists of a variety of immunoglobulin proteins, the most prominent of which is Fel $\mathrm{d} 1$. This allergen accounts for $60-90 \%$ of total allergenicity. The majority of production of this protein occurs in the sebaceous glands of the animal. It is transferred to the skin through the sebum, where it accumulates in the fur and can then be released into the environment. When inhaled by humans, Fel d 1 can induce an allergic response in susceptible individuals. Due to the complexity of this allergen, a completely effective treatment has yet to be developed. Therefore, methods to reduce the production and release of Fel $\mathrm{d} 1$ in the cat should be explored. Sebum production is under regulation of many hormones and mediators, and nutritional compounds may act as inhibitors in some pathways. By inhibiting certain points in these pathways with nutritional compounds such as polyphenols, carotenoids, fatty acids, and a decreased glycemic index diet, it may be possible to decrease production of Fel d 1 in cats. With a lower release of the allergen and adequate cleaning management, there is a high probability based on the current hypothesis to lower its concentration in the environment and consequently, decrease the symptoms in allergic people.
\end{abstract}

Abbreviations: AA : Arachidonic Acid; AR : Androgen receptor; DHT : Dihydrotestosterone; Fel d1: Felisdomesticus 1; IGF-1 I : Insulin-like growth factor; PPAR : Peroxisome proliferator activated receptor; SIT : Specific immunotherapy; LA: linoleic acid

\section{Introduction}

Over the years, the relationship between humans and their pets has evolved to the point that these animals have become a member of the family. Cats are one of the most popular pets worldwide. In the United States alone, 42.9 million households own a cat, accounting for a total cat population of 85.8 million [1]. Although owning a pet can have both physical and mental health benefits, many pet owners suffer from an allergy triggered by a protein produced by many pets, including cats. Close contact with pets leads to an enhanced ambient exposure to their allergens, resulting in an increase in the prevalence of human allergy to pet dander [2]. Symptoms from pet allergies can range from rhinoconjunctivitis to potentially life-threatening asthmatic exacerbations, and proteins produced by the domestic cat are some of the most potent elicitors of allergic disease [3]. The prevalence of sensitization to cats varies between countries and regions depending on the cultural differences and environmental factors that may affect the human-cat relationship [4]. It was revealed through skin prick tests that $8.8 \%$ and $17 \%$ of the European and US population are sensitized to cat dander [5]. While some owners decide to keep their cat and suffer through the ownership process with over-the-counter medications or take allergy shots to de-sensitize themselves, others choose not to keep their pets at all. As a result, even though a cat's allergenicity does not have a direct impact on the animals health, it is a concern regarding public health, as allergies to cats are the second most commonly reported cause of cat relinquishment [6].
Studies have shown that the major cat allergen, a protein produced mainly in sebaceous glands, is found in high concentrations in public spaces due its aerodynamic properties. Because of this, avoidance of cats does not necessarily guarantee a life without allergy crisis. Therefore, decreasing cats' allergenicity by controlling its allergen production is a more effective and lasting way to control the allergy. There is anecdotal evidence that cats fed certain foods might be less provoking, and understanding the physiology behind the allergens production and how they trigger immune response are essential to discovering how a diet might be modified to decrease the cat's allergenicity.

This current review will explore the existing literature regarding the immune response and clinical manifestation of cat allergy, and what steps are taken currently to address the issue through medical intervention and other means. Further, it is the intent of this review to understand how cat dander becomes the offending antigenic agent, what might be done at a basic level of ownership, and what modifies the production of the offending antigen, focusing mainly on possible dietary interventions.

\section{Cat Allergens}

Allergens are proteins, or chemicals bound to proteins, that elicit immediate hypersensitivity reactions in an atopic individual who is chronically exposed [7]. Cat dander, which refers to particles that are sloughed from animal skin, contains several allergens, and currently eight of them have been identified and described: Felis domesticus (Fel d 1; secretoglobulin), Fel d 2 
(albumin), Fel d 3 (cystatin), Fel d 4 (salivary lipocalin), Fel d 5w (IgA), Fel D 6w (IgM), Fel d 7 (von Ebner gland protein) and Fel d 8 (latherin-like protein) [2,8]. Among all the known allergens present in cat dander, Fel $\mathrm{d} 1$ has been identified as the major contributor to allergies, accounting for $60-90 \%$ of the total allergenicity of cat dander [9].

Fel $\mathrm{d} 1$ is a uteroglobin-like protein included in the secretoglobulin family, and it is formed by the fusion of two 18$20 \mathrm{kD}$ heterodimers, each of which is composed of two disulphidelinked peptide chains: chain 1 with 70 amino acids and chain 2 with 90-92 amino acids [10,11]. Despite the limited sequence identity, the structure of Fel $\mathrm{d} 1$ shows a striking similarity to that of uteroglobin, a protein member of the secretoglobin family that may carry ligands such as steroids [12]. It has been found that each Fel $\mathrm{d} 1$ heterodimer has a cavity that is capable of interacting with ligands such as testosterone, progesterone and polychlorinated biphenyl (PCB), a steroid analog, like uteroglobin. Furthermore, it has been reported that the Fel $\mathrm{d} 1$ molecule is $\mathrm{N}$-glycolasylated and has three putative calcium binding sites, which may play a role in the immune response [13].

Although it has been found that Fel d 1 production occurs in multiple glands throughout the body, such as lachrymal and salivary glands, its major site of production is in the sebaceous glands $[14,15]$. Fel $\mathrm{d} 1$ is produced by the intradermal hair follicle and is released to the skin using the sebum as a carrier. Once on the skin, the allergen accumulates on the fur, which serves as a reservoir for the allergen. Though the cat is able to spread the allergen throughout the fur during grooming and cleaning, anatomical sites with higher concentrations of sebaceous glands have higher accumulation of Fel $\mathrm{d} 1$ in the fur, such as the face [16]. It has also been reported that Fel $\mathrm{d} 1$ concentrations are higher in the neck than the chest, back, stomach, and haunches areas [17]. Anal glands, which are composed of large amounts of sebaceous glands, also have high concentrations of Fel d 1 [18]. Secretions from anal glands have a distinctive odor and are released by frightened cats, possibly as a communication tool. Cats that are "handling-avoidant" and present aggressive behaviors have a greater release of Fel d 1 compared to those presenting a shy and/or friendly behavior, suggesting that aggressive cats may express their anal sacs during unwanted handling [19].

A single cat can produce anywhere from 3-7 $\mu$ g of Fel d 1 each day [14]. This production varies not only between cats but also within the same cat, throughout the span of one day and/or between multiple days. Moreover, it is also influenced by the cats' sex $[19,20]$. Male cats produce greater amounts of the allergen than females, as sebum production is directly dependent on androgens, mainly testosterone. Castrated males have a decreased metabolic activity in the sebaceous glands, due to decreased testosterone, and consequently they produce less Fel d 1 compared to intact males. However, castrated males still produce Fel d 1 in sufficient concentration to trigger allergy responses. The negative effect of castration on the cellular activity and Fel d 1 production is totally reversed with testosterone treatment, showing that both the sebaceous glands and Fel $\mathrm{d} 1$ output are under hormonal control [21].

Although the specific physiologic role of Fel $\mathrm{d} 1$ is still unknown, findings thus far offer some support for its understanding. The close association of Fel d 1 with skin sebaceous and anal glands, its steroid-binding property, and the correlation between the allergen and cat behavior suggests that Fel $\mathrm{d} 1$ may act as a carrier protein for molecules involved in the communication between species. Furthermore, Fel d 1 is present in high concentrations on the face, an anatomical site known to produce pheromones of familiarization [16]. Adding to possible function as a carrier, Fel d 1 may also play a role in the regulation of lipids on skin and cat fur, as it is produced at sites with a high rate of lipid secretion [18].

\section{Immune response to Fel d 1}

The human respiratory system is constantly exposed to a great number of inhaled particles of inorganic and organic origin [22]. Some of these particles, such as the cat allergen Fel d 1, can induce allergic responses in susceptible individuals. An allergic reaction happens in two steps: first exposure (sensitization) and then a subsequent exposure. Fel d 1 comes into contact with the immune system by inhalation, and can bind to the mannose receptor through its cysteine-rich domain in dendritic cells (DC). Dendritic cells are professional antigen-presenting cells, meaning that they constitutively express the major histocompatibility complex class II molecule [9]. These cells process the allergen and present its epitopes (peptides of the protein allergen that specifically trigger the immune system) to the CD4+ T cells, also called $\mathrm{T}$ helper cells (Th). Thus far, only four Fel $\mathrm{d} 1$ epitopes have been discovered. Increasing information on the epitopes is important for understanding the allergen immune response and cross-reactivity, as well as to improve the current available treatments [23]. It has been reported that there is some crossreactivity between some cat and dog allergens. This property is related to the similarity of the molecules. The more similar they are, the greater the chance of cross-reactivity. Fel $d 4$ and Can $f$ 6 , a dog allergen, showed cross-reactivity in addition to cat and dog albumin [24,25]. An allergen in dog dander has also been detected that cross-reacts with Fel $d$. However, this specific allergen has not yet been described, and further studies are needed to characterize its clinical relevance [26].

When inhaled, Fel d 1 can trigger three different patterns of humoral responses in humans: 1) the allergic response, which is characterized by the activation of Thelper 2 (Th2) and production of IgE and IgG to Fel d 1;2) a modified Th2 response, in which there is a production of IgG4 and absence of IgE; and 3) the absence of humoral response to Fel d 1 [27]. In allergic people, naïve Th differentiate into Th2, which induce immunoglobulin class switch in B-cells to the antibody IgE. Th2 cells are not only important for IgE production, they also produce many cytokines that are able to directly stimulate the appearance of allergic symptoms [28]. The allergen-specific IgE binds to its high affinity receptor present in 
basophils and mast cells. In a subsequent exposure, these mast cells use their coated IgE structure to bind to Fel d 1, leading to degranulation and triggering histamine release. Histamine is one of the chemical compounds responsible for common allergy symptoms. Fel d 1 also enhances tool-like receptor activity by lipid ligands, activating innate immune signaling pathways [29].

In non-allergic individuals, due to environmental, genetic, and other unknown reasons, naïve Th differentiates into $\mathrm{T}$ helper 1 (Th1), which is not able to stimulate IgE production [28]. There are two main theories that attempt to explain the relationship between cats and allergic disease: the allergen hypothesis and the hygiene hypothesis [30]. Per the allergen hypothesis, increased exposure to allergens leads to increased allergic disease. However, it has been shown that children raised in houses with cats are less likely to become allergic to them, contradicting this theory [31]. Recent studies reported that allergic people who are chronically exposed to cat allergens have a modified Th2 response that switches the production of IgE to IgG4 in B-cells, driving to desensitization [32].

Alternatively, the hygiene hypothesis suggests that there is a shift from Th1 predominance response to Th2 due to a reduced exposure to childhood parasitic, bacterial, and viral infections [2]. Although not all studies have found this relationship, some of them have shown a lower prevalence of atopy in children living on farms with direct contact to animals in comparison to those children who did not live the same rural lifestyle. Children with higher contact with animals were most likely to have higher exposure to products of bacteria, such as lipopolysaccharide $[33,34]$.

\section{Treatment}

Due to the complexity of an allergic response, a desirable treatment has still not been fully developed. Allergen-specific immunotherapy (SIT) has been used most frequently over the years. This therapy works by inducing tolerance to the allergen via allergen exposure and preventing the progression of allergic diseases. However, this treatment has drawbacks such as the treatment persistence and the possibility of inducing mild to severe adverse reactions. Side effects are possibly mediated by the allergen-specific $\mathrm{T}$ cells and the production of allergenspecific IgE antibodies [35].

In order to reduce allergenicity and enhance SIT treatment safety, different routes of administration and strategies for improving SIT have been investigated, such as targeting the allergen to Fcy receptors creating a chimeric human-cat fusion protein, and linking allergens to $\mathrm{CpG}$-containing nucleotides or carbohydrate-based particles $[36,37,38]$. Most of the new approaches are in clinical studies and not available to the market yet.

The characterization of Fel d 1 structure, as well as the identification of its epitopes has contributed to improving SIT. However, the commercial extracts available for SIT still have great variability in their allergen contents [35]. Further studies are needed to better understand which specific components generate the immune response, and to explore the results of possible treatments in clinical trials to evaluate their safety and efficacy [35].

Each individual reacts differently to SIT and its avoidance is recommended to patients with severe and threating adverse reactions. As not all individuals can benefit from SIT, decreasing the allergen concentration in the environment is an important step to control one's allergy response. Feeding the cat a less provoking diet might be a practical and convenient method to accomplish this.

\section{Epidemiology}

Controlling exposure to cat allergens is of great importance in reducing the risk of sensitization, as well as for managing symptomatic allergic individuals [39]. The first approach recommended by doctors to allergic people owning cats is to get rid of them. Consequently, a cat's allergenicity has a public health (and cat) implications, as it can increase the relinquishment of pets. Although houses with a cat have higher concentrations of Fel $\mathrm{d} 1$ in comparison to houses without, Fel $\mathrm{d} 1$ may also be found in houses without a cat [40]. This offending allergen is a very heatstable protein and can cling to various materials and surfaces in the house, such as walls, carpets and furniture. So, even after the cat removal, it can take many months before the levels of Fel $\mathrm{d} 1$ in dust reservoirs of the home significantly fall $[30,41]$. In addition, Fel $\mathrm{d} 1$ is carried by particles that range from less than $1 \mu \mathrm{m}$ to greater than $20 \mu \mathrm{m}$ in mean diameter; these small particles are able to stay airborne for long periods of time after disturbance, making them easily inhaled [42].

Furthermore, indirect exposure to the cats' allergens can occur. Based on its aerodynamic characteristics and its ability to "stick" to all available surfaces, Fel d 1 can be transferred to places that have never been occupied by cats, such as schools, hospitals, and day-care centers, and can be found in concentrations high enough to activate symptoms and sensitize individuals $[42,43,44]$. The allergen can also be carried by other means, such as clothes, making it very easy to transport from one location to another [45].

There is no scientific evidence to support the concept of hypoallergenic cats [46]. Therefore, the only possibility for effectively decreasing the number of people sensitized to cat dander is by decreasing the output of the antigens in the environment. Washing the cat has been thought to be a successful approach; however, while washing of cat reduces the level of Fel d 1 greatly on cat skin and fur, the original level is restored within 2 days [16]. There is anecdotal evidence that cats fed certain foods might be less provoking allergenically. Building off of these anecdotes, there may be specific ingredients that can interfere with, and decrease the production of Fel $\mathrm{d} 1$. Therefore, it is of great importance to understand the physiology behind the production of Fel d 1 in order to attempt to create a diet capable of controlling a cat's allergen production. 


\section{Nutritional Approach}

It has been reported that many of the cat's tissues and secretions are capable of producing Fel $d$. However, the main site of production of the allergen is the sebaceous glands [18]. Studies have shown that a decrease in the sebaceous glands activity and, consequently, a decrease in sebum production lead to a lower Fel d 1 output [21]. Focusing the efforts on understanding the sebaceous glands physiology and how it can affect Fel d 1 production can be an effective way to significantly decrease its output in the environment [Figure 3].

Sebaceous glands are holocrine glands formed by clusters of specialized cells called the sebocyte. They are located in the dermis of the skin, and are found all over the body. The majority of these glands are associated with the hair follicle, forming the pillosebaceous unit, but they can also be found without an associated hair follicle [47], such as in the anal glands of cats. Furthermore, they are not located uniformly over the body and are in higher concentration in specific anatomical sites [16].

The main function of mature sebaceous glands is the production of sebum, a complex of lipids, through holocrine rupture of individual sebocytes [48]. Sebum composition varies greatly among species, but mostly consists of cholesterol, fatty acids, fatty alcohols, di- and tri-glycerides, wax esters, sterol esters, and squalene [49]. However, the specific composition of the cats' sebum has not yet been reported. Although the putative function of the sebum has not been completely revealed, it may act as a delivery system for antioxidants, antimicrobial lipids, and pheromones [47]. This supports the hypothesis that Fel $\mathrm{d} 1$ acts as a pheromone-carrier protein. In addition, it can be hypothesized that Fel $\mathrm{d} 1$ binds to the pheromone that is produced in the sebaceous glands, and the complex is released with the sebum to the skin.

Although sebum production by sebaceous glands seems to be a simple and straightforward process, it is under the regulation of innumerous mechanisms. It is a target, and also a source, of many endocrine hormones and autocrine/paracrine mediators [50]. It has been confirmed that Fel d 1 concentration on the skin is under hormonal control in parallel with the sebaceous glands [21]. Some of these hormones and mediators are affected by, and can be modified by dietary factors. The main mechanisms by which dietary factors can affect the sebaceous glands, and consequently the Fel d 1 output will be further discussed below.

\section{Androgens}

Androgens are steroidal hormones that are mainly produced by the gonads and the adrenal gland, and can also be produced within the sebaceous glands. Sebum production is under the control of androgens, mainly testosterone and DHT. Sebaceous glands may be characterized as a secondary sexual organ due the direct effect of androgens on sebum production [20]. As Fel d 1 production originates in these glands, its production is also influenced by androgens.
The effects of testosterone and androgens on sebaceous gland activity are mediated by binding to the nuclear androgen receptor (AR) expressed in sebocytes. Although testosterone can bind to the AR, DHT is a much more potent androgen with a greater affinity to the receptor [47]. In addition, sebocytes express the enzyme $5 \alpha$-reductase that reduces testosterone to DHT [51]. Once DHT binds to the AR, it stimulates the proliferation of sebocytes and increases lipid production through the transcript factor sterol-response element binding protein (SREBP) [47].

The effects of androgens on sebaceous glands can be reduced by either inhibiting the $5 \alpha$-reductase activity or directly decreasing the hormone production, as observed in castrated animals. Male cats have a significant decrease in testosterone production after castration, and consequently, a decrease in sebocyte metabolic activity.

Some dietary compounds are known to have the ability to influence androgen production [52]. Polyphenols are antioxidant molecules found in many foods, and a variety of them are known to have the ability to inhibit the $5 \alpha$-reductase enzyme. Specific isoflavones from soybeans have also been identified as efficient inhibitors. It has also been reported that rats fed with a phytoestrogen-rich diet, containing around $600 \mu \mathrm{g}$ isoflavones, presented plasma testosterone levels approximately $50 \%$ lower in comparison to rats fed a phytoestrogen-free diet [53]. Polyphenols may also directly reduce lipogenesis through SREBP-1 pathway thereby decreasing sebum production [54].

Interestingly enough compounds found in tomatoes may also affect androgen production. In humans, elevated consumption of tomato products is linked to reduced risk of prostate cancer [55]. Even though cats lack the prostate gland, tomato products may block androgen production at the same level as in humans. The main components of tomatoes associated with androgen production is the non-provitamin A carotenoid lycopene. It has been reported that short-term carotenoid intake has a negative effect on serum testosterone concentration [56].

No studies have been conducted evaluating the effect of polyphenols and carotenoids on androgen and Fel d 1 production in cats. However, based on previous studies it can be hypothesized that those dietary components may reduce the allergen output by decreasing androgen production.

Another form of vitamin A that is a potent inhibitor of sebum production is 13-cis retinoic-acid, which is widely used for acne treatment in humans. Its mechanism of action is not known. 13cis retinoic-acid may serve as a pro-drug for synthesis of all trans retinoic acid that in fact interact with the retinoic receptors and decrease sebaceous gland activity [51]. However, 13-cis retinoicacid is teratogenic, so it does not seem to be the best approach to decrease sebum production in cats.

\section{Peroxisome proliferator activated receptors}

Sebaceous glands are not only under the regulation of hormones, but are also controlled by paracrine and autocrine 
lipid mediators [50]. Peroxisome proliferator activated receptors (PPARs) are orphan nuclear receptors that form heterodimers with retinoid $\mathrm{X}$ receptors (RXR) and binding to specific response elements on DNA [47]. These receptors are expressed in human skin and in sebaceous glands where they possibly mediate sebum production by inducing particular gene expression.

There are three subtypes of PPARs: $\alpha, \delta$, and $\gamma$ with PPAR $\gamma$ being the most expressed in sebocytes. Ligands for PPARs can be either synthetic or natural, such as fatty acids [51,57]. Linoleic acid (LA) is a natural ligand and has been demonstrated to increase lipid production and differentiation of sebocytes. Although it is still not known if arachidonic acid (AA) binds to PPAR, it also promotes these same responses [58,59]. It has also been reported that androgen hormones only modify lipid synthesis in cultured sebocytes in the presence of certain co-activators, such as LA [60]. Furthermore, the differentiation-promoting AA is able to stimulate PPARs as well as its derivative leukotriene (LTB4), an inflammatory molecule [61]. Therefore, LA and AA, which are both classified as omega- 6 fatty acids, are able to induce sebum production in sebocytes by binding to PPARs or by the stimulation of inflammatory molecules that are able to trigger that response [Figure 1].

A cat diet with a high omega-6/omega-3 (n6:n3) ratio may stimulate sebum and Fel d 1 production. Modifying this ratio by adding ingredients with a greater concentration of omega-3 fatty acids may induce a lesser (reduced) inflammatory status in the animal and, thereby a lower production of Fel $d$ 1. It is worth remembering that polyunsaturated fatty acids are more susceptible to oxidation. So, it is essential to not only formulate a diet that has the desired ratio of n6:n3, but also guarantee the stability of those fatty acids to meet the cat's requirement and to take advantage of their potential nutraceutical properties.

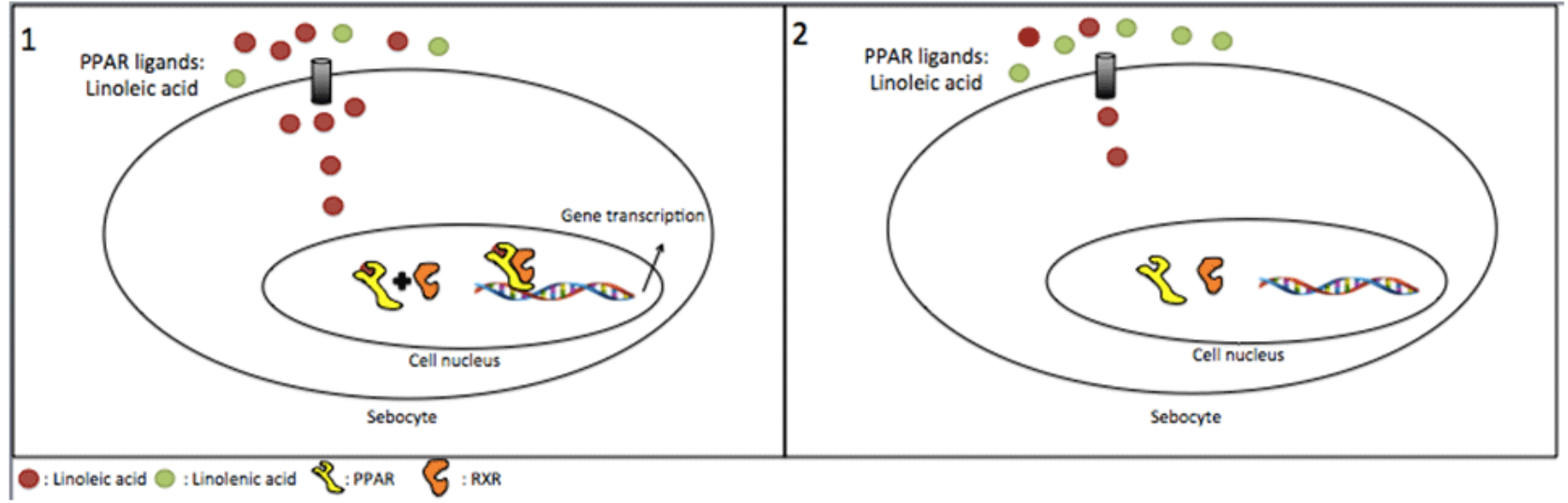

Figure 1: Possible effect of diets containing different n6:n3 ratio in sebum production. A diet with high n6:n3 ratio induces a higher PPAR response; 2- A diet with lower n6-n3 ratio do not induce a PPAR response so frequently as it has a lower proportion of its natural ligand, linoleic acid

\section{High glycemic-index diet}

It has been reported in humans that a western diet, characterized by high glycemic load and high dairy protein consumption, is an important nutritional factor that promotes acne. This diet leads to an increase in insulin and insulin - like growth factor (IGF-1) serum levels, which stimulate sebum production [62]. Insulin alone induces hepatic IGF-1 secretion, and both hormones stimulate lipid production in sebocytes. IGF-1 markedly increases sebocytes proliferation, while insulin stimulates both proliferation and differentiation of sebocytes. These hormones stimulate the PI3K/AKT pathway, which in turn induce SREBP-1 production and other genes that have an influence on lipid production and sebocyte function [63]. Furthermore, the PI3K/AKT represses the nuclear transcription factor FoxO1, which suppresses androgen receptor, and PPARs [62]. Also, hyperinsulinemia promotes androgen stimulation to adrenals, testes as well as the ovaries, increasing the effect of androgen on sebum production [64] [Figure 2].
A diet with a low glycemic index leads to a lower level of insulin production, and, subsequently, IGF-1 production. Cats are obligate carnivores and therefore have no obligate requirement for carbohydrates which are often included in cat diets for both processing and cost considerations. Therefore, a low glycemic index diet, low in carbohydrates, would be a logical strategy for decreasing sebum production and Fel d 1 output in cats.

\section{Conclusions}

The human-cat relationship has been changing over the past 5 to 6 decades and has reached a point to which they have become a part of the family. Although having a pet brings many health and emotional benefits, the allergic reactions that many people face can be highly detrimental. An effective treatment for these allergies has not yet been developed. The major cat allergen is Fel d 1, a protein produced mainly in sebaceous glands. Its function has not been fully discovered, but recent findings strongly suggest that it acts as a carrier for pheromones. Due its aerodynamic properties, Fel d 1 can stick to many different 


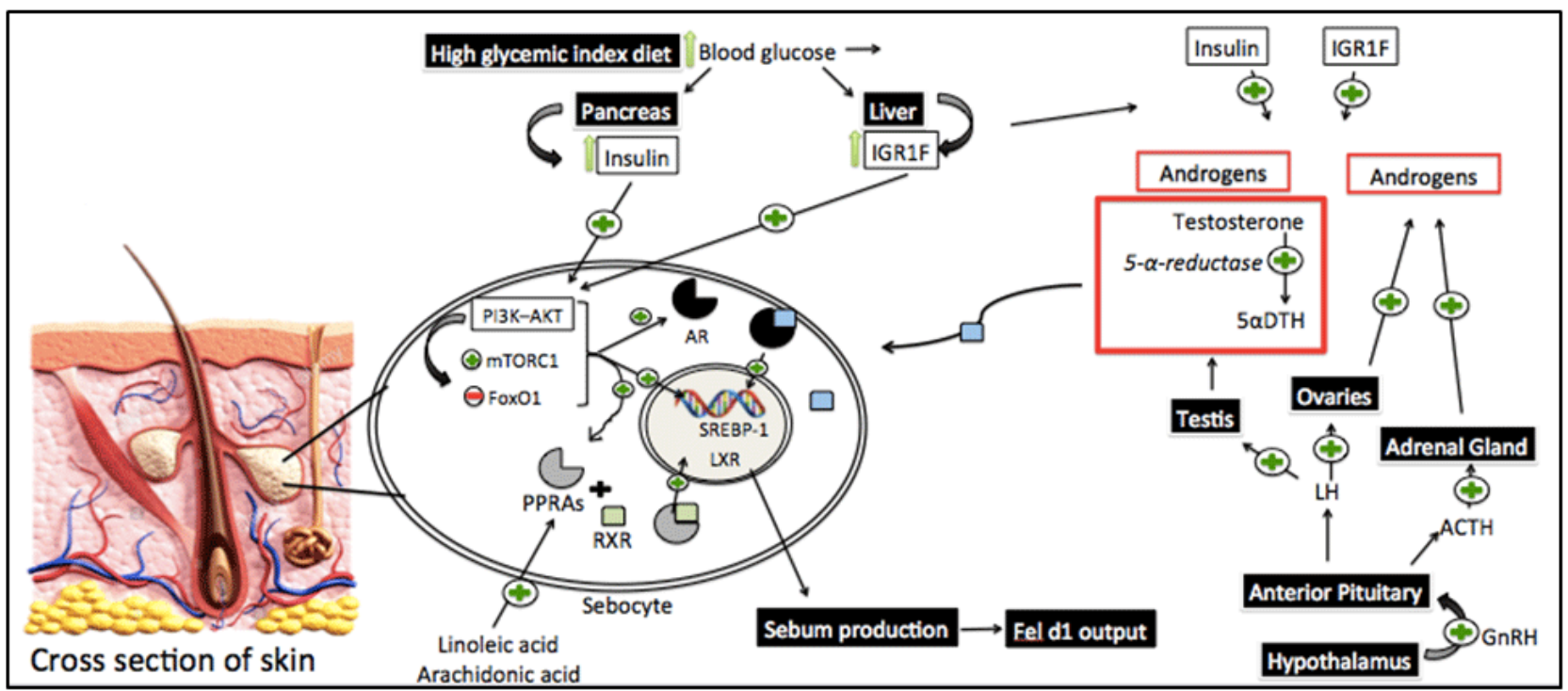

Figure 2: Control of sebocyte activity by some mediators

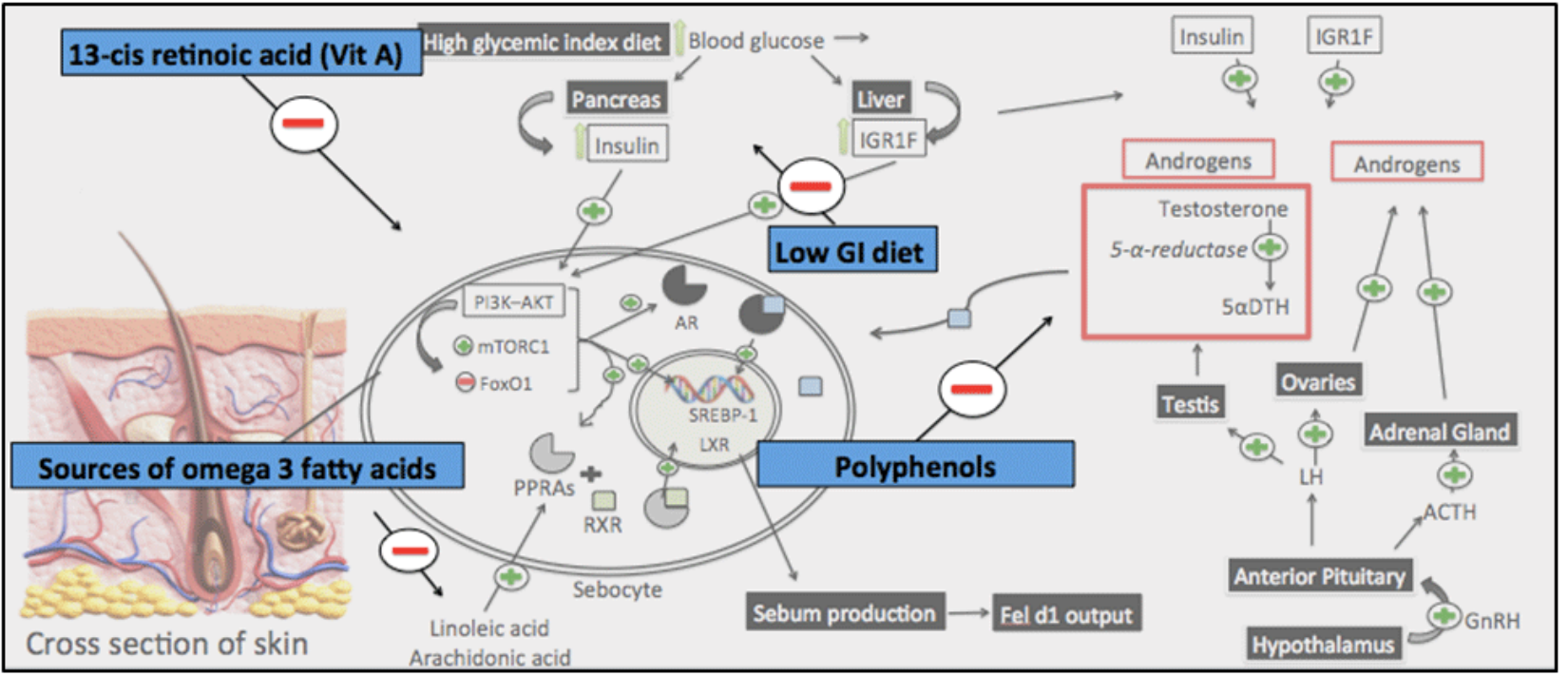

Figure 3: Nutritional components that can interfere in sebocyte activity

objects and is ubiquitous in the environment.

Decreasing the output of Fel $\mathrm{d} 1$ may be a practical way to reduce allergy symptoms in sensitized people. Sebum acts as a carrier of Fel $d$, and its reduction is strongly correlated with a decrease in Fel d 1 output. Sebum production is under the regulation of many hormones and mediators, and nutritional compounds may act as inhibitors in some pathways. Further study evaluations of such nutritional compounds are needed. A diet with a low glycemic index, high levels of polyphenols and vitamin $A$, and an adequate $n 6: n 3$ ratio may be a potential less provoking diet to cats [Figure 3].

It is important to point out that allergies to cats involve environmental and physiological factors involving both humans and the animals. A less provoking diet will not act as treatment for allergic reactions in humans but may be a practical way to decrease Fel $\mathrm{d} 1$ output to aid in the management of the condition. With a lower release of the allergen and adequate cleaning management, there is a high probability based on the current hypothesis to lower its concentration in the environment, and, consequently, decrease the symptoms in allergic people. 


\section{References}

1. APPA National Pet Owners Survey Debut. Julie Springer. 2017-2018

2. Daniel O. Morris. Human allergy to environmental pet danders: A public health perspective. Vet Dermatol. 2010;21(5):441-449. doi:10.1111/j.1365-3164.2010.00882.x.

3. Gronlund H, Adedoyin J, Reininger R, E M Varga, M. Zach, M. Fredriksson, et al. Higher immunoglobulin E antibody levels to recombinant Fel d 1 in cat-allergic children with asthma compared with rhinoconjunctivitis. ClinExp Allergy. 2008;38(8): 1275-1281. doi: 10.1111/j.1365-2222.2008.03003.x.

4. Eva Zahradnik, Monika Raulf. Animal allergens and their presence in the environment. Front Immunol. 2014;5(76):1-21. doi: 10.3389/ fimmu.2014.00076

5. Oliver Pfaar, Ludger Klimek, Eva Maria Varga. Fel d 1 synthetic peptides (Cat-PAD) - Good news for cat owners with children? Pediatr Allergy Immunol. 2016;27(7):666-670. doi: 10.1111/pai.12602

6. Stenoroden K, Morris K, Olson P. U.S. Pet (Dog and Cat) Population Fact Sheet.

7. Abul Abbas Andrew H. Lichtman Shiv Pillai. Immunologic tolerance and autoimmunity In: Abbas AK, Lichtman $\mathrm{AH}$, Pillai $\mathrm{S}$, editors. Cellular and molecular immunology. 7th ed.Philadelphia, PA: Elsevier Saunders. 2012;319-344.

8. Smith W, O Neil SE, Hales BJ, Chai TLY, Hazell L A, Tanyaratsrisakul S, et al. Two newly identified cat allergens: The von Ebner gland protein Fel $\mathrm{d} 7$ and the latherin-like protein Fel d 8. Int Arch Allergy Immunol. 2011;156(2):159-170. doi: 10.1159/000322879

9. Mohamed Emara, Pierre Joseph Royer, Zaigham Abbas, Herb F Sewell, Gihan Gebriel Mohamed, Sonali Singh,et al. Recognition of the major cat allergen fel d 1 through the cysteine-rich domain of the mannose receptor determines its Allergenicity. J Biol Chem. 2011;286(15):13033-13040. doi: 10.1074/jbc.M111.220657

10. Oscar A.Duffort, JoséCarreira, Gianpaolo Nitti, Florentino Polo, Manuel Lombardero. Studies on the biochemical structure of the major cat allergen Felis domesticus. Mol Immunol. 1991;28(4-5):301-309.

11. LiccardiG, D amato G, Russo M, Canonica G.W, D Amato L, De Martino M, et al. Focus on cat allergen (Fel d 1): Immunological and Aerodynamic Characteristics, Modality of Airway Sensitization and Avoidance strategies. Int Arch Allergy Immunol. 2003;132(1): 1-12. doi: 10.1159/000073259

12. Liselotte Kaiser, Hans Gronlund, Tatyana Sandalova, Hans Gustaf Ljunggren, Marianne van Hage-Hamsten, Adnane Achour, et al. The crystal structure of the Major Cat Allergen Fel D 1, A Member of the Secretoglobin. Family J Biol Chem. 2003;278(39): 37730-37735.

13. Rodrigo Ligabue-Braun, Liana Guimarães Sachett, Laércio Pol-Fachin, Hugo Verli. The calcium Goes Meow: Effects of ions and Glycosylation on Fel d 1, the major Cat Allergen. PLoS One. 2015;10(7):1-19. doi: 10.1371/journal.pone.0132311.

14.Dabrowski AJ, Van Der Brempt X, Soler M, N Seguret, P Lucciani, D.Charpin, D.Vervloet, et al. Cat skin as an important source of Fel d I Allergen. J Allergy Clin Immunol. 1990;86(4):462-465.
15. Charpin C, Mata P, Charpin D, Lavaut MN, Allasia C, Vervloet D.Fel d 1 allergen distribution in car fur and skin. J Allergy Clin Immunol. 1991;88(1):77-82.

16. Carayol N, Birnbaum J, Magnan A, M. Ramadour, A. Lanteaume, D. Vervloet, et al. Fel d 1 production in the cat skin varies according to anatomical sites. Allergy. 2000;55(6):570-573. doi: 10.1034/j.13989995.2000.00588.x

17.David B Avner, Matthew S Perzanowski, Thomas A E Platts Mills, Judith A. Woodfolk. Evaluation of different techniques for washing cats: quantification of allergens removed from the cat and the effect on airboneFel d 1. J Allergy Clin Immunol. 1997;100(3):307-312. doi:10.1016/S0091-6749(97)70242-2

18. De Andrade AD, Birnbaum J, Magalon C, J P Magnol, A Lanteaume, D Charpin, et al. Fel d 1 levels in cat anal glands. ClinExp Allergy. 1996;36(4):432-434. doi: 10.1111/j.1365-2222.1996.tb00077.x

19. Cecile Bienboire Frosiniac, Alessandro Cozzia, Céline Lafont Lecuelle, Daniel Vervloet, Catherine Ronin, Patrick Pageat. Immunological differences in the global release of the major cat allergen Fel d 1 are influenced by sex and behaviour. Vet J. 2012;193(1):162-167. doi: 10.1016/j.tvjl.2011.09.031

20. Jose Jalil-Colome, Armele Dornelas de Andrade, Joelle Birnbaum, Denise Casanova, Jean-Louis Mege, Andre Lanteaume, et al. Sex difference in Fel d 1 allergen production. J Allergy Clin Immunol.1996;98(1):165-168. doi:https://doi.org/10.1016/S00916749(96)70238-5

21. Charpin C, Zielonka TM, Charpin D, Ansaldi JL, Allasia C, Vervloet D. Effects of castration and testosterone on Fel d I productionby sebaceous glands of male cats: II morphometric assessment.ClinExpAllergy. 1994;24(12):1174-1178. doi: 10.1111/j.1365-2222.1994.tb03325.x.

22. Hentges F, Leonard C, Arumugam K, Hilger C. Immune responses to inhalant mammalian allergens. Front Immunol. 2014;5(234):1-8. doi: 10.3389/fimmu.2014.00234

23. Natt Tasaniyananda, Anchalee Tungtrongchitr, Watee Seesuay, Yuwaporn Sakolvaree, Nitaya Indrawattana, Wanpen Chaicumpa, et al. A novel IgE-binding epitope of cat major allergen, Fel d 1. BiochemBiophys Res Commun. 2016;470(3):593-598. doi: 10.1016/j. bbrc.2016.01.099.

24. Nilsson OB, Binnmyr J, Zoltowska A, Saarne T, van Hage M, Gronlund H. Characterization of the dog lipocalin allergen Can $f$ 6: the role in cross-reactivity with cat and horse. Allergy. 2012;67(6):751-757. doi: 10.1111/j.1398-9995.2012.02826.x

25. Cabañas R, Lopez-Serrano MC, Carreira J, Ventas P, Polo F, Caballero M T. Importance of albumin in cross-reactivity among cat, dog and horse allergens. J InvestigAllergolClin Immunol. 2000;10(2):71-77.

26. Reininger R, Varga EM, Zach M, Balic N, Lindemeier AD, Swoboda I, et al. Detection of an allergen in dog dander that cross-reacts with the major allergen,Fel d 1. ClinExp Allergy. 2007;37(1):116-124. doi: 10.1111/j.1365-2222.2006.02611.x

27.Amanda J Reefer, Raquel M Carneiro, Natalie J Custis, Thomas AE Platts Mills, Sun Sang J Sung, Juergen Hammer, et al. A Role for 
IL-10-mediated HLA-DR7-restricted $\mathrm{T}$ cell-dependent events in development of the modified Th2 response to cat allergen. J Immunol. 2004;172(5):2763-2772. doi: 10.4049/jimmunol.172.5.2763

28. Sergio Romagnani. T-cell subsets (Th1 versus Th2). Ann Allergy Asthma Immunol. 2000;85(1):9-21. doi: 10.1016/S1081-1206(10)62426-X.

29. Jurgen Herre, Hans Gronlund, Heather Brooks, Lee Hopkins, Lisa Waggoner, Ben Murton, et al. Allergens as immunomodulatory proteins: the cat dander protein Fel d 1 enhances TLR activation by lipid ligands. J Immunol. 2013;191(4):1529-1535. doi: 10.4049/ jimmunol.1300284

30. Dharmage SC, Lodge CL, Matheson MC, Campbell B, Lowe AJ. Exposure to cats: Update on risks for sensitization and allergic diseases.Curr Allergy Asthma Rep. 2012;12(5):413-423. doi: 10.1007/s11882-0120288-x.

31. Hesselmar B, Aberg N, Aberg B, Eriksson B, Bjorksten B. Does early exposure to cat or dog protect against latter allergy development? Clin Exp Allergy. 1999;29(5):611-617. doi: 10.1046/j.13652222.1999.00534.x.

32. Renand A, Archila LD, McGinty J, Erik Wambre, David Robinson, Belinda J Hales, et al. Chronic cat-allergen exposure induces a TH2 celldependent IgG4 response related to low-sensitization. J Allergy Clin Immunol. 2015;136(6):1627-1635. doi: 10.1016/j.jaci.2015.07.031.

33. Kilpelainen M, Terho, EO, Helenius H, Koskenvuo M. Farm environment in childhood prevents the development of allergies. ClinExp Allergy. 2000;30(2):201-2018. doi: 10.1046/j.1365-2222.2000.00800.x.

34. Von EhrensteinOS,Von Mutius E, llli S, Baumann L, Bohm O, von Kries R. Reduced risk of hay fever and asthma among children of farmers.ClinExp Allergy. 2000;30(2):187-193. doi: 10.1046/j.13652222.2000.00801.x.

35.Van Hage M, Pauli G. New vaccines for mammalian allergy using molecular approaches, Front Immunol. 2014;5:81. doi: 10.3389/ fimmu.2014.00081

36.Zhu D, Kepley CL, Zhang K, Terada T, Yamada T, Saxon A. A chimeric human-cat fusion protein blocks cat-induced allergy. Nat Med. 2005;11(4):446-449. doi: 10.1038/nm1219

37. Santeliz JV, Van Nest G, Traquina P, Larsen E, Wills-Karp M. Amb a 1-linked CpG oligo-deoxynucleotides reserve established airway hyperresponsiveness in a murine model of asthma. J Allergy Clin Immunol. 2002;109(3):455-462. doi: 10.1067/mai.2002.122156

38. Neimert-Andersson T, Thunberg S, Swedin L, Wiedermann U, Jacobsson Ekman G, Dahlén SE, et al. Carbohydrate-based particles reduce allergic inflammation in a mouse model for cat allergy. Allergy.2008;63(5):518-526. doi:10.1111/j.1398-9995.2008.01644.x.

39. De Blay F, Chapman MD, Platts Mills TA. Airborne cat allergen (Fel d I). Environmental control with the cat in situ. Am Rev Respir Dis. 1991;143(6):1334-1339. doi: 10.1164/ajrccm/143.6.1334.

40. Custovic A, Simpson A, Pahdi H, Green RM, Chapman MD, Woodcock A. Distribution, aerodynamic characteristics, and removal of the major cat allergen Fel d 1 in British homes. Thorax. 1998;53(1):33-38. doi: 10.1136/thx.53.1.33
41. Wood RA, Chapman MD, AdkinsonNF Jr, Eggleston PA. The effect of cat removal on allergen content in the household dust samples. J Allergy Clin Immunol. 1989;83(4):730-734.

42. Wood RA, Laheri AN, Eggleston PA. The aerodynamic characteristics of cat allergen.ClinExp Allergy. 1993;23(9):733-739. doi: 10.1111/ j.1365-2222.1993.tb00360.x.

43. Neal JS, Arlian LG, Morgan MS. Relationship among house-dust mites, Der 1 , Fel $\mathrm{d} 1$, and Can $\mathrm{f} 1$ on clothing and automobile seats with respect to densities in houses. Ann Allergy Asthma Immunol. 2002;88(4):410415. doi: 10.1016/S1081-1206(10)62373-3.

44. Munir AKM, Einarsson R, Shou C, Dreborg S. The amount of the major car (Fel d 1) and dogs (Can f 1) allergens in dust from Swedish schools in high enough to probably cause perennial symptoms in most children with asthma who are sensitized to cat and dog. J Allergy Clin Immunol. 1993;91:1067:1074.

45. Gennaro D Amato, Gennaro Liccardi, Maria Russo, Domingo Barber, Maria D Amato, Jose Carreira. Clothing is a carrier of cat allergens. J Allergy Clin Immunol. 1997;99(4):577-578. doi: 10.1016/S00916749(97)70088-5.

46. Ahmed Butt, Daanish Rashid, Richard F. Lockey. Do hypoallergenic cats and dogs exist? Ann Allergy Asthma Immunol. 2012;108(2):74-76. doi: 10.1016/j.anai.2011.12.005

47. Smith KR, Thiboutot DM. Thematic review series: Skin Lipids. Sebaceous gland lipids: friend or foe? J Lipid Res. 2008, 49(2):271281. doi: 10.1194/jlr.R700015-JLR200

48. Picardo M, Ottaviani M, Camera E, Mastrofrancesco A. Sebaceous glands lipids. Dermatoendocrinol. 2009;1(2):68-71.

49. Stewart ME, Downing DT. Chemistry and Function of mammalian sebaceous lipids. Adv Lipid Res. 1991;24:263:301. doi: 10.1016/ B978-0-12-024924-4.50013-4.

50. Toth B, Olah A, SzollosiAG, Czifra G, Biro T. “Sebocytes' makeup” - Novel mechanisms and concepts in the physiology of the human sebaceous glands. Pflugers Arch. 2011;461(6):593-606. doi: 10.1007/s00424011-0941-6.

51. Thiboutot D. Regulationof Human Sebaceous Glands. J Invest Dermatol. 2004;123(1):1-12. doi: 10.1111/j.1523-1747.2004.t01-2-.x.

52. Mijeong Bae, Mijin Woo, Irawan Wijaya Kusuma, Enos Tangke Arung, Chae Ha Yang, Yong ung Kim. Inhibitory Effects of Isoflavonoids on Rat Prostate Testosterone $5 \alpha$-Reductase. J Acupunct Meridian Stud.2012;5(6):319-322.doi: 10.1016/j.jams.2012.07.022.

53. Weber KS, Setchell KD, Stocco DM, Lephart ED. Dietary soyphytoestrogens decrease testosterone levels and prostate weight without altering $\mathrm{LH}$, prostate 5alfa-reductase or testicular steroidogenic acute regulatory peptide levels in adult male SpragueDawley rats. J Endocrinol. 2001;170(3):591-599.

54. Ji YoungYoon, Hyuck HoonKwon, Seong Uk Min, Diane M Thiboutot, Dae HunSuh. Epigallocatechin-3-gallate improves acne in humans by modulating intracellular molecular targets and inhibiting. J Invest Dermatol. 2013;133(2):429-440. doi: 10.1038/jid.2012.292.

55. Giovannucci E, Ascherio A, Rimm EB, Stampfer MJ, Colditz GA, Willett 
WC. Intake of carotenoids and retinol in relation to risk of prostate cancer. J Natl Cancer Inst. 1995;87(23):1767-1776. doi: 10.1093/ jnci/87.23.1767.

56. Campbell JK, Stroud CK, Nakamura MT, Lila MA, Erdman JW Jr. Serum testosterone is reduced following short-term phytofluene, lycopene, or tomato powder consumption in F344 rats. J Nutr. 2006;136(11):28132819.

57. Kliewer SA, Lehmann JM, Willson TM. Orphan nuclear receptors: shifting endocrinology into reverse. Science. 1999;284(5415):757760. doi:10.1126/science.284.5415.757

58. Chen W, Yang CC, Sheu HM, Seltmann H, Zouboulis CC. Expression of peroxisome proliferator-activated receptor and CCAAT/enhancer binding protein transcription factors in cultured human sebocytes. J Invest Dermatol. 2003;121(3):441-447. doi: 10.1046/j.15231747.2003.12411.x.

59. Anna Wrobel, Holger Seltmann, Sabine Fimmel, Karin Müller Decker, Miki Tsukada, Birgit Bogdanoff, et al. Differentiation and apoptosis in human immortalized sebocytes. J Invest Dermatol. 2003;120(2):175181. doi: 10.1046/j.1523-1747.2003.12029.x.
60. Rosenfield RL, Deplewski D, Kentsis A, Ciletti N. Mechanisms of androgen induction of sebocyte differentiation. Dermatology. 1996;196(1):43-46.

61. Devchand PR, Keller H, Peters JM, Vazquez M, Gonzalez FJ, Wahli W. The PPARalpha-leukotriene B4 pathway to inflammation control. Nature. 1996;384(6604):39-43. doi: 0.1038/384039a0.

62. Bodo C Melnik, Christos C Zouboulis. Potentital role of Fox01 and mTORC1 in the pathogenesis of Western diet-induced acne. ExpDermatol. 2013;22(5): 311-315. doi: 10.1111/exd.12142.

63. Megha Kataria Arora, AmitaYadav, Vandana Saini. Role of hormones in acne vulgaris. ClinBiochem. 2011;44(13):1035-1040. doi: 10.1016/j. clinbiochem.2011.06.984

64. Rashmi Kumari, Devinder Mohan Thappa. Role of insulin resistance and diet in acne. Indian J DermatolVenereolLeprol. 2013;79(3): 291299. doi:10.4103/0378-6323.110753 